\title{
Positive ALDH1A3 and Negative GPX3 Expressions Are Biomarkers for Poor Prognosis of Gallbladder Cancer
}

\author{
Zhu-lin Yang, ${ }^{1}$ Leping Yang, ${ }^{1}$ Qiong Zou, ${ }^{2}$ Yuan Yuan, ${ }^{2}$ Jinghe $\mathrm{Li}^{3}{ }^{3}$ Lufeng Liang, \\ Guixiang Zeng, ${ }^{5}$ and Senlin Chen ${ }^{6}$ \\ ${ }^{1}$ Research Laboratory of Hepatobiliary Diseases, Second Xiangya Hospital, Central South University, Changsha, \\ Hunan 410011, China \\ ${ }^{2}$ Department of Pathology, Third Xiangya Hospital, Changsha, Hunan 410013, China \\ ${ }^{3}$ Department of Pathology, Xiangya Medical School, Central South University, Changsha, Hunan 410011, China \\ ${ }^{4}$ Department of Hepatobiliary and Pancreatic Surgery, Hunan Provincial People's Hospital, Changsha, Hunan 410007, China \\ ${ }^{5}$ Department of Pathology, Loudi Central Hospital, Loudi, Hunan 417011, China \\ ${ }^{6}$ Department of Pathology, Hunan Provincial Tumor Hospital, Changsha, Hunan 410013, China
}

Correspondence should be addressed to Zhu-lin Yang; zhulinyang@yahoo.com

Received 14 April 2013; Accepted 28 June 2013

Academic Editor: Holly Soares

Copyright (C) 2013 Zhu-lin Yang et al. This is an open access article distributed under the Creative Commons Attribution License, which permits unrestricted use, distribution, and reproduction in any medium, provided the original work is properly cited.

Background. Gallbladder cancers (GBCs) are highly aggressive cancers with high mortality. However, biological markers for the progression and prognosis of GBC are currently unavailable in the clinic. Objective. To identify biomarkers for predicting GBC metastasis and prognosis. Methods. We examined ALDH1A3 and GPX3 expressions in 46 squamous cell/adenosquamous carcinomas (SC/ASC) and 80 adenocarcinomas (AC) by using immunohistochemistry. Results. Positive ALDH1A3 and negative GPX3 expressions were significantly associated with lymph node metastasis and invasion of SC/ASCs and ACs. Univariate KaplanMeier analysis showed that either positive ALDH1A3 $(P<0.001)$ or negative GPX3 $(P<0.001)$ expression significantly correlated with decreased overall survival in both SC/ASC and AC patients. Multivariate Cox regression analysis showed that positive ALDH1A3 expression or negative GPX3 expression was an independent poor-prognostic predictor in both SC/ASC and AC patients. Conclusions. Our study suggested that positive ALDH1A3 and negative GPX3 expressions are closely associated with clinical pathological behaviors and poor prognosis of gallbladder cancer.

\section{Introduction}

Gallbladder cancers (GBCs) are highly aggressive cancers with the highest mortality rate among gastrointestinal cancers. Most patients die within a year of diagnosis. The highest prevalence rates of GBC have been observed in American Indian, Chilean, and Japanese women [1,2]. Early diagnosis of GBC is generally impossible due to a lack of specific signs or symptoms. Over $90 \%$ of GBC patients are diagnosed at an advanced, inoperable stage with serious invasion and metastasis to other organs [3]. A majority of GBCs encountered in the clinic are adenocarcinomas (AC, 90-95\%) [4], while rare subtypes such as squamous cell/adenosquamous carcinoma (SC/ASC), mucinous carcinoma, signet ring cell carcinoma, and undifferentiated carcinoma are hardly ever encountered $[2,5]$. At present, cholecystectomy is only viable for early stage GBC [6-8], while patients with unresectable or metastatic GBC have extremely poor prognosis and few treatment options. Generally, palliative chemotherapy and radiation therapy offer limited benefits to patients with GBC [2]. Therefore, there is an urgent need to identify biological markers for the diagnosis, prognosis, and target therapy of gallbladder cancers.

Numerous studies have observed elevated levels of oxidative stress during tumor growth, which is characterized by an increase in reactive oxygen species (ROS). ROS serve as secondary messenger molecules to increase tumor cell proliferation, genetic mutations, and instability, which lead to subsequent invasion, angiogenesis, and drug resistance in 
cancer cells [9]. Most importantly, cancer cells can develop mechanisms to evade high ROS-induced apoptosis $[10,11]$. The elevation of oxidative stress levels in tumor cells is generally thought to be associated with two factors: the increased generation of ROS and the decreased capacity of the cell to eliminate ROS [12]. Enhanced release of $\mathrm{O}_{2}{ }^{-{ }^{-}}$and/or $\mathrm{H}_{2} \mathrm{O}_{2}$ from the mitochondria and activation of NADPH oxidase (NOX) systems increase cellular ROS levels $[13,14]$. The increase in ROS can also be caused by the suppression of antioxidant enzymes, such as MnSOD and GPX [7]. Thus, the differential expression of antioxidant enzymes in cancer can serve as biomarkers of tumor initiation and/or progression.

Tumor suppressive roles of the superoxide dismutase (SOD) family of proteins have been observed in a variety of cancers. However, how SOD is regulated remains unclear. Glutathione peroxidase (GPX) has been revealed to modulate the tumor suppressive effect of MnSOD in various tumor cells [12]. A number of studies have demonstrated that GPX3 expression is downregulated in a variety of cancers. For example, GPX3 is strongly downregulated in prostate cancer, thyroid cancer, colorectal cancer, gastric cancer, and breast cancer $[15,16]$. Intriguingly, a previous study has revealed that GPX3 expression is higher in clear cell epithelial ovarian carcinoma tissues compared to control tissues [17]. This might suggest that GPX3 activity is tumor-specific. Since GPX3 is always highly expressed in healthy tissues, it has been suggested to be a tumor suppressor in many tumors [18]. However, the expression of GPX3 in gallbladder cancers has not been reported.

Aldehyde dehydrogenases (ALDHs) are a family of enzymes that metabolize both endogenous and exogenous aldehydes to carboxylic acids as well as other reactive compounds [19]. ALDHs can function as antioxidants. For example, ALDH1A1 and ALDH3A1 function as ocular antioxidants that play unique roles in the defense of the eye against UV radiation and in the downstream effects of oxidative stress [20]. Some ALDH isoforms function in retinoic acid (RA) signaling via RA production by oxidizing all-transretinal and 9-cis-retinal [21]. Recently, ALDH1 activity has been employed as a marker of stem-like cells in many cancers, such as cervical and breast cancer [22]. Moreover, ALDH1 activity was demonstrated to be significantly higher in metastatic breast tumor cells that escaped chemotherapy [23]. Furthermore, high activity of ALDH1 is associated with poor prognosis in breast, bladder, and prostate cancer patients $[24,25]$. However, most ALDH prognostic data is based on using ALDH1A1 expression levels as an indicator for cancer patients' outcome $[26,27]$. These studies also yielded varied results, and the correlation of ALDH1A1 with prognosis may be dependent on cancer type [21]. In contrast, ALDH1A3, but not ALDH1A1, has been shown to correlate consistently with tumor grade and metastasis of breast cancer patients [28], suggesting that ALDH1A3 may be a better marker in some tumors than ALDH1A1. The association of other ALDH subtypes with tumor progression and prognosis has not been established.

Our preliminary study using microarray assay showed a 24-fold increase in ALDH1A3 level and 23-fold decrease in GPX3 in GBC-SD cells compared to normal GBC epithelial cells (data not shown). In this study, the expression of ALDH1A3 and GPX3 in surgically resected specimens, including AC and SC/ASC, was examined by immunohistochemistry. The correlation of ALDH1A3 and GPX3 expressions with the clinicopathological characteristics and prognosis of AC and SC/ASC was comparatively evaluated.

\section{Materials and Methods}

2.1. Case Selection. A total of 46 squamous cell/adenosquamous carcinomas (SC/ASC) from GBC patients that underwent surgical resection or biopsy were collected from January 1995 to December 2009. The percentage of SC/ASC in various gallbladder cancers is $4.34 \%$ ( $46 / 1060 \mathrm{GBCs}) .80$ adenocarcinomas (AC) samples for patients that underwent surgical resection or biopsy were collected from January 2005 to December 2009. All diagnoses were based on morphological criteria, immunohistochemical staining, and clinical findings. A gallbladder cancer sample was diagnosed as SC when most malignant cells are squamous cells and less than $10 \%$ are adenocarcinoma cells. A gallbladder cancer sample was diagnosed as ASC when the tumor contains both squamous cells and adenocarcinoma cells, but the tumor must contain at least $10 \%$ adenocarcinoma or squamous cell carcinoma cells. Among the 46 SC/ASC patients, 19 patients were male and 27 patients were female with an age variation from 35 to $82(55.8 \pm 9.6)$ years. Among the $80 \mathrm{AC}$ patients, 26 patients were male and 54 patients were female with an age variation from 33 to $80(53.8 \pm 9.9)$ years. The differentiation (refer to squamous cells) of the 46 SC/ASCs includes 16 well-differentiated, 24 moderately differentiated, and 6 poorly differentiated carcinomas. Among the 80 ACs, 27 were well differentiated, 25 were moderately differentiated, and 28 were poorly differentiated. Among the 46 SC/ASC patients, invasion of gallbladder, surrounding tissues and organs, was found in 30 patients, while 29 patients had regional lymph node metastasis. Among the $80 \mathrm{AC}$ patients, invasion of gallbladder surrounding tissues and organs was found in 49 patients, while 50 patients had regional lymph node metastasis. Surgery included radical resection for 14 SC/ASCs and 26 ACs, palliative surgery for 18 SC/ASCs and $28 \mathrm{ACs}$, and no operation for 14 SC/ASCs and 26 ACs with only biopsy. The 2-year survival information of $80 \mathrm{AC}$ and 46 SC/ASC patients was obtained through letters and phone calls. This study was preapproved by the Ethics Committee for Human Research, Central South University.

2.2. Immunohistochemistry Staining. Four-micrometer-thick sections were cut from routinely paraffin-embedded tissues of AC and SC/ASC. Staining was conducted with the peroxidase-based EnVision Detection kit (Dako Laboratories, CA, USA) by following the user manual and the rabbit anti-ALDH1A3 (Abgent Company, California, USA) and rabbit anti-GPX3 (Novus Biologicals, Littleton, USA) antibodies which specifically stain human ALDH1A3 and human GPX3. Briefly, the sections were deparaffinized and then incubated with $3 \% \mathrm{H}_{2} \mathrm{O}_{2}$ for 15 minutes. After being soaked with phosphate-buffered saline (PBS) for $3 \times 5$ minutes, 
the sections were incubated with primary antibody for $1 \mathrm{hr}$ at room temperature. After rinsing sections with PBS for 3 times, solution A was added, and the sections were incubated for 30 minutes. The substrate DAB was added followed by hematoxylin counterstaining. The slides were dehydrated with different concentrations $(70 \%-100 \%)$ of alcohol and soaked in xylene for $3 \times 5$ minutes. ALDH1A3 and GPX3 positive cells exhibited brown cytoplasmic staining. The positive control was the positive biopsy provided by Beijing Zhongshan Biotechnology Company (Beijing, China), while the negative control was designed by replacing the primary antibody with $5 \%$ fetal bovine serum. By considering that heterogeneity of staining pattern exists, the percentage of positive cells was calculated from 500 tumor cells in 10 random fields. The staining strength was graded as previously described [29]: a score of 1 was given for no positive staining or a uncertainly weak staining; a score of 2 was given for weak to moderate staining; a score of 3 was given for moderate to strong staining. For GPX3, cases with both positive cells $\geq$ $25 \%$ and scores $\geq 2$ were considered positive. A few cases with $10 \%$ to $25 \%$ positive cells and 3 scores in staining were also regarded as positive. For ALDH1A3, cases with both positive cells $\geq 10 \%$ and scores $\geq 2$ were considered positive.

Image analysis was performed as previously described [30]. The slides were systematically scanned, and 7-8 representative digital images were acquired from each slide. Digital images were quantified by image analysis with Adobe Photoshop, version 7.0 (Adobe Systems, San Jose, CA), and the extent of staining was assessed as the percentage of positively stained area per field under $\times 100$ magnification.

2.3. Statistical Analysis. Data was analyzed using the statistical package for the Social Sciences Version 13.0 (SPSS 13.0). The interrelationship of ALDH1A3 or GPX3 expressions with histological or clinical factors was analyzed using $\chi^{2}$, Fisher's exact test, or Student's $t$-test. Kaplan-Meier and time series tests (log-rank test) were used for univariate survival analysis. Cox proportional hazards model was used for multivariate analysis and to determine the $95 \%$ confidence interval.

\section{Results}

3.1. Comparison of Clinicopathological Characteristics and ALDH1A3 and GPX3 Expressions between SC/ASC and AC. The percentage of cases with age older than 45 years and tumor mass $>3 \mathrm{~cm}$ was significantly higher in SC/ASCs than in ACs $(P<0.05)$. The percentage of cases with poorly differentiated tumor was significantly lower in SC/ASCs than in ACs. No significant differences in other clinicopathological characteristics were observed between SC/ASC and AC patients. EnVision immunohistochemistry revealed that ALDH1A3 and GPX3 positive reaction was mainly localized in the cytoplasm of tumor cells in SC/ASC (Figure 1) and AC (Figure 2), but a small amount of inflammatory reaction cells and fibroblasts was also stained. western blot with ALDH1A3 antibody showed one main band of $56 \mathrm{kDa}$ and a weak band of $22 \mathrm{kDa}$, while Western blot with GPX3 antibody showed one band of $25 \mathrm{kDa}$ (data not shown). No significant difference in the percentage of cases with positive ALDH1A3 and GPX3 expressions was observed between SC/ASC and AC patients (data not shown).

3.2. The Association of ALDH1A3 and GPX3 Expressions with Clinicopathological Characteristics of Patients with SC/ASC and $A C$. As shown in Table 1 and Figure 3(a), the percentage of cases with positive ALDH1A3 expression was significantly higher in tumor tissues from SC/ASC patients with high TNM stage and lymph node metastasis compared to tumor tissues from cases with low TNM stage and no lymph metastasis $(P<0.05$ or $P<0.01)$. The GPX3 expression was significantly lower in cases with higher TNM stage, lymph node metastasis, and invasion $(P<0.05$ or $P<0.01)$. ALDH1A3 and GPX3 exhibited no significant association with other clinicopathological characteristics of SC/ASC.

The percentage of cases with positive ALDH1A3 expression and negative GPX3 expression in AC tumors was significantly higher in cases with poor differentiation, large tumor mass, high TNM stage with lymph node metastasis, and invasion to the gallbladder surrounding tissues and organs compared to the cases with well-differentiated tumor, small tumor mass, lower TNM stage, no lymph metastasis, and no invasion $(P<0.05, P<0.01$, or $P<0.001)$ (Table 1 , Figure 3(b)).

\subsection{The Correlation between ALDH1A3 or GPX3 Expressions} with Survival in Patients with SC/ASC and AC. Survival information of $46 \mathrm{SC} / \mathrm{ASC}$ patients was obtained through letters and phone calls. The followup time was 2 years, and patients that survived longer than 2 years were included in the analysis as censored cases. Thirty-three of the 46 SC/ASC patients survived less than 1 year and 13 patients survived over 1 year ( 4 cases survived longer than 2 years) with an average survival time of $10.07 \pm 0.78$ months. Survival information of 80 AC patients was obtained. Fifty-seven of the $80 \mathrm{AC}$ patients survived less than 1 year and 23 patients survived over 1 year ( 9 cases survived longer than 2 years) with an average survival time of $10.34 \pm 0.63$ months.

The Kaplan-Meier survival analysis in SC/ASC patients revealed that the differentiation, tumor size, TNM stage, lymph node metastasis, and invasion $(P<0.001)$ were significantly associated with the average survival time (Table 2 ). The average survival time of ALDH1A3 positive patients was significantly lower than patients with negative ALDH1A3 expression $(P=0.005)$, but the average survival time of GPX3 positive patients was significantly higher than patients with negative GPX3 expression $(P=0.002)$ (Table 2, Figure 4$)$. Cox multivariate analysis showed that the differentiation, tumor size $(\geq 3 \mathrm{~cm})$, TNM stage, invasion, and operative procedure as well as ALDH1A3-positive expression or GPX3negative expression were negatively correlated with postoperative survival, suggesting that ALDH1A3 positivity is a risk factor and GPX3 has a strong impact on prognosis of SCs/ASCs (Table 3).

The Kaplan-Meier survival analysis in AC patients revealed that the differentiation, tumor size, TNM stage, lymph node metastasis, invasion, and operative procedure 

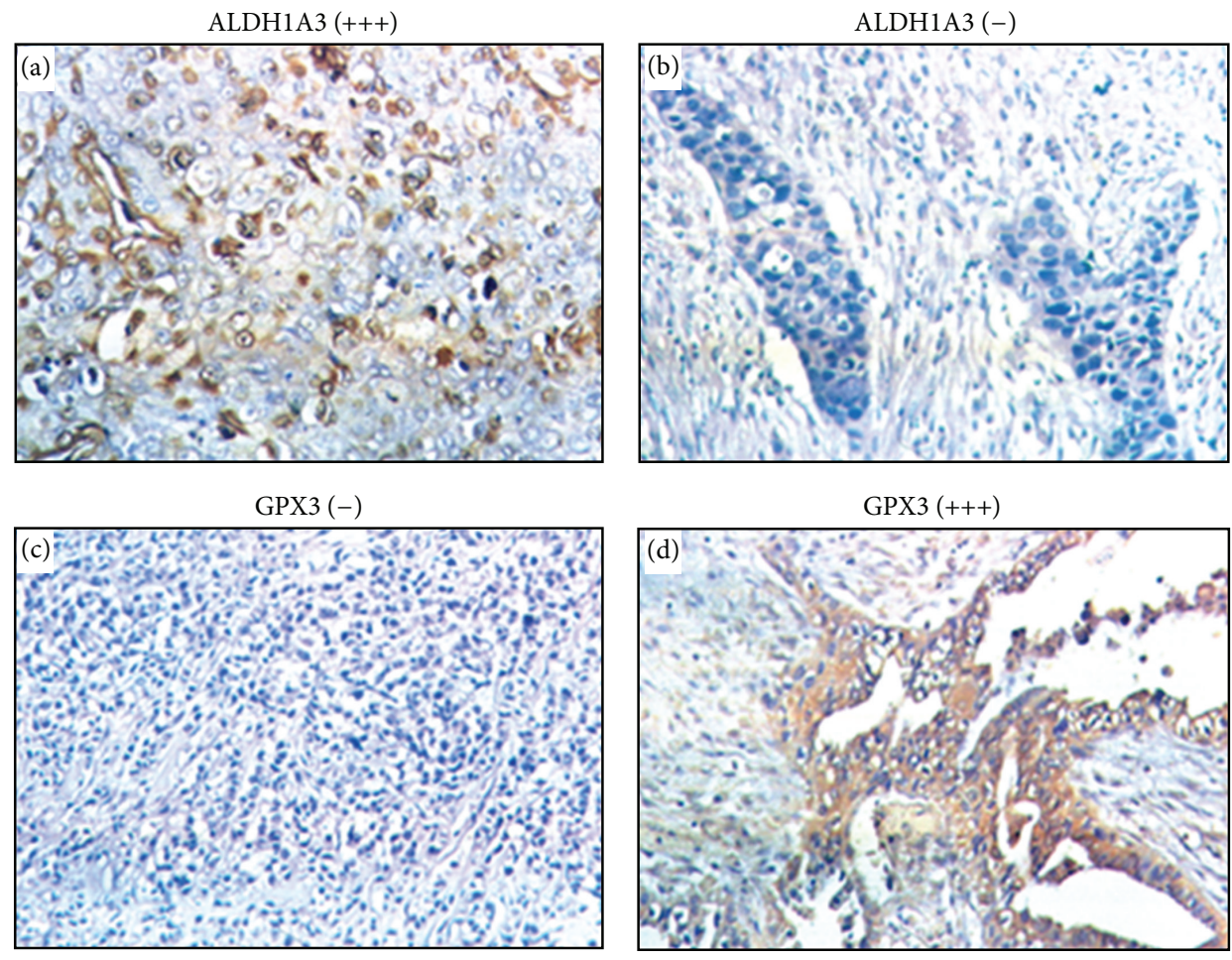

FIGURE 1: ALDH1A3 and GPX3 expressions in squamous carcinomas/adenosquamous carcinomas (SC/ASC). EnVision immunohistochemistry, original magnification $\times 200$. ALDH1A3 and GPX3 positive reaction was mainly localized in the cytoplasm. (a), positive ALDH1A3 expression in poorly differentiated SC/ASC. (b), negative ALDH1A3 expression in well-differentiated SC/ASC. (c), negative GPX3 expression in poorly differentiated SC/ASC. (d), positive GPX3 expression in well-differentiated SC/ASC.
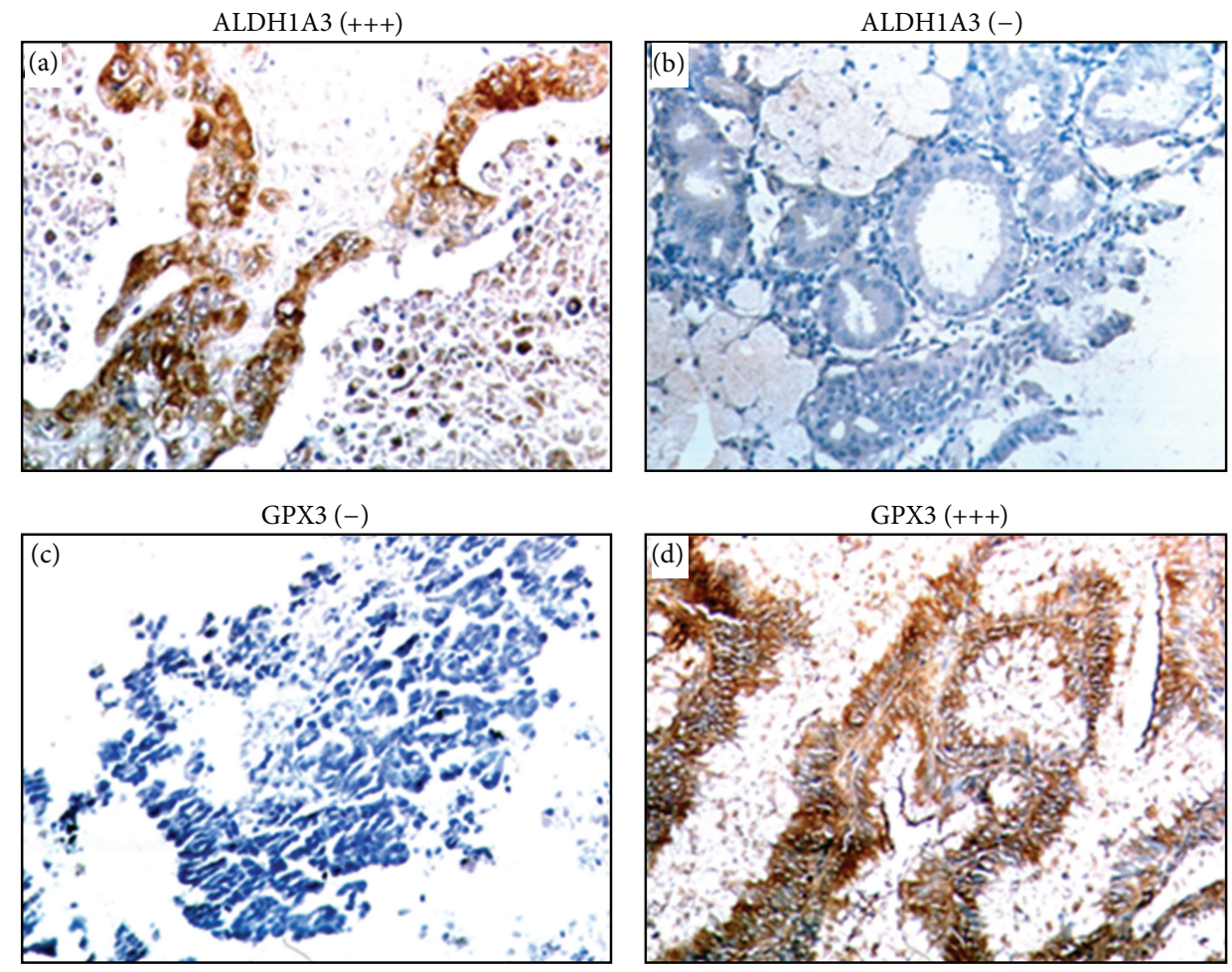

FIGURE 2: ALDH1A3 and GPX3 expressions in adenocarcinoma (AC). EnVision immunohistochemistry, original magnification $\times 200$. ALDH1A3 and GPX3 positive reaction was mainly localized in the cytoplasm. (a), positive ALDH1A3 expression in moderately differentiated AC. (b), negative ALDH1A3 expression in well-differentiated AC. (c), negative GPX3 expression in poorly differentiated AC. (d), positive GPX3 expression in well-differentiated AC. 
TABLE 1: The association of ALDH1A3 and GPX3 expressions with the clinicopathological characteristics of SC/ASC and AC.

\begin{tabular}{|c|c|c|c|c|c|c|c|}
\hline \multirow{2}{*}{ Clinicopathological characteristics } & \multirow{2}{*}{ Total number } & \multicolumn{3}{|c|}{ ALDH1A3 } & \multirow{2}{*}{\multicolumn{2}{|c|}{$\begin{array}{c}\text { GPX3 } \\
\text { Pos number }(\%)\end{array}$}} & \multirow[b]{2}{*}{$\chi^{2}$} \\
\hline & & Pos number (\%) & $\chi^{2}$ & $P$ & & & \\
\hline \multicolumn{8}{|l|}{ SC/ASC } \\
\hline \multicolumn{8}{|l|}{ Differentiation } \\
\hline Well & 16 & $5(31.3)$ & \multirow{3}{*}{3.111} & \multirow{3}{*}{0.153} & $8(50.0)$ & \multirow{3}{*}{2.001} & \multirow{3}{*}{0.352} \\
\hline Moderately & 24 & $7(29.2)$ & & & $10(41.7)$ & & \\
\hline Poorly & 6 & $4(66.7)$ & & & $1(16.7)$ & & \\
\hline \multicolumn{8}{|l|}{ Tumor mass size } \\
\hline$\leq 3 \mathrm{~cm}$ & 20 & $4(20.0)$ & \multirow{2}{*}{3.409} & \multirow{2}{*}{0.065} & $10(50.0)$ & \multirow[b]{2}{*}{1.104} & \multirow[b]{2}{*}{0.293} \\
\hline$>3 \mathrm{~cm}$ & 26 & $12(46.2)$ & & & $9(34.6)$ & & \\
\hline \multicolumn{8}{|l|}{ TNM stage } \\
\hline $\mathrm{I}+\mathrm{II}$ & 12 & $2(16.7)$ & \multirow{3}{*}{7.953} & \multirow{3}{*}{0.019} & $8(66.7)$ & \multirow{3}{*}{7.512} & \multirow{3}{*}{0.021} \\
\hline III & 20 & $5(25.0)$ & & & $9(45.0)$ & & \\
\hline IV & 14 & $9(64.3)$ & & & $2(14.3)$ & & \\
\hline \multicolumn{8}{|l|}{ Lymph metastasis } \\
\hline No & 17 & $2(11.8)$ & \multirow{2}{*}{6.298} & \multirow{2}{*}{0.016} & $11(64.7)$ & \multirow{2}{*}{6.091} & \multirow{2}{*}{0.019} \\
\hline Yes & 29 & $14(48.3)$ & & & $8(27.6)$ & & \\
\hline Invasion & & & & & & & \\
\hline No & 16 & $3(18.8)$ & & & $10(62.5)$ & & \\
\hline Yes & 30 & $13(43.3)$ & 2.780 & 0.091 & $9(30.0)$ & 4.546 & 0.031 \\
\hline $\mathrm{AC}$ & & & & & & & \\
\hline Differentiation & & & & & & & \\
\hline Well & 27 & $6(22.2)$ & & & $18(66.7)$ & & \\
\hline Moderately & 25 & $10(40.0)$ & 11.840 & 0.003 & $13(52.0)$ & 9.865 & 0.007 \\
\hline Poorly & 28 & $19(67.9)$ & & & $7(25.0)$ & & \\
\hline Tumor mass size & & & & & & & \\
\hline$\leq 3 \mathrm{~cm}$ & 50 & $15(30.0)$ & & & $29(58.0)$ & & \\
\hline$>3 \mathrm{~cm}$ & 30 & $20(66.7)$ & 10.243 & 0.001 & $9(30.0)$ & 5.895 & 0.015 \\
\hline TNM stage & & & & & & & \\
\hline $\mathrm{I}+\mathrm{II}$ & 21 & $6(28.6)$ & & & $17(81.0)$ & & \\
\hline III & 38 & $15(39.5)$ & 6.728 & 0.039 & $17(44.7)$ & 16.357 & 0.000 \\
\hline IV & 21 & $14(66.7)$ & & & $4(19.1)$ & & \\
\hline Lymph metastasis & & & & & & & \\
\hline No & 30 & $7(23.3)$ & 8130 & 0004 & $20(66.7)$ & 7071 & 0008 \\
\hline Yes & 50 & $28(56.0)$ & 8.130 & 0.004 & $18(36.0)$ & 7.071 & 0.008 \\
\hline Invasion & & & & & & & \\
\hline No & 31 & $9(29.0)$ & & & $21(67.7)$ & & \\
\hline Yes & 49 & $26(53.1)$ & 4.455 & 0.035 & $17(34.7)$ & 8.316 & 0.003 \\
\hline
\end{tabular}

$(P<0.001)$ were significantly associated with the average survival time (Table 2 ). The average survival time of ALDH1A3-positive AC patients was significantly lower than patients with negative ALDH1A3 expression $(P<0.001)$, but the average survival time of GPX3 positive patients was significantly higher than patients with negative GPX3 expression $(P<0.001)$ (Table 2 , Figure 5). Cox multivariate analysis showed that the differentiation, tumor size $(\geq 3 \mathrm{~cm})$, TNM stage, lymph node metastasis, invasion, and operative procedure as well as ALDH1A3-positive expression or GPX3negative expression were negatively correlated with poor survival of AC patients (Table 3).

\section{Discussion}

The clinical and pathological characteristics of squamous cell/adenosquamous carcinomas (SC/ASC) of the gallbladder have not yet been widely recognized because these subtypes are rather rare. Moreover, no study so far has systemically compared the clinical, pathological, and molecular differences between the rare SC/ASC subtype and the common AC subtype of gallbladder cancers (GBC). In this study, no significant differences in differentiation, metastasis, invasion, TNM stage, and prognosis were observed between these two subtypes. The clinicopathological presentations of SC/ASC did not seem to be different from ordinary AC though more $\mathrm{SC} / \mathrm{ASC}$ patients exhibited larger tumor mass $(>3 \mathrm{~cm})$ than AC patients. This suggested that squamous differentiation is as aggressive as glandular differentiation in the gallbladder, which is inconsistent with previous observations [31, 32]. Importantly, we found no differences in ALDH1A3 and GPX3 expressions between SC/ASC and AC patients, suggesting that they may have similar biological characteristics.

Numerous studies have observed an increase in oxidative stress during tumor growth, including GBC [33]. The GPX proteins are antioxidant enzymes and ALDH proteins 
TABLE 2: Relationship between ALDH1A3 and GPX3 expressions, clinicopathological characteristics, and average survival of SC/ASC and AC patients.

\begin{tabular}{|c|c|c|c|c|}
\hline C.P characteristics & Samples $(n)$ & Average survival (month) & Chi-square & $P$ value \\
\hline \multicolumn{5}{|l|}{ SC/ASC } \\
\hline \multicolumn{5}{|l|}{ Differentiation } \\
\hline Well & 16 & $13.81(5-24)$ & \multirow{3}{*}{19.125} & \multirow{3}{*}{0.000} \\
\hline Moderately & 24 & $8.92(4-18)$ & & \\
\hline Poorly & 6 & $5.83(4-9)$ & & \\
\hline \multicolumn{5}{|l|}{ Tumor mass size } \\
\hline$\leq 3 \mathrm{~cm}$ & 20 & $14.35(7-24)$ & \multirow{2}{*}{31.337} & \multirow{2}{*}{0.000} \\
\hline$>3 \mathrm{~cm}$ & 26 & $7.04(4-11)$ & & \\
\hline \multicolumn{5}{|l|}{ TNM stage } \\
\hline $\mathrm{I}+\mathrm{II}$ & 12 & $17.00(9-24)$ & \multirow{3}{*}{51.139} & \multirow{3}{*}{0.000} \\
\hline III & 20 & $9.20(7-15)$ & & \\
\hline IV & 14 & $5.86(4-8)$ & & \\
\hline \multicolumn{5}{|c|}{ Lymph node metastasis } \\
\hline No & 17 & $14.24(4-24)$ & \multirow{2}{*}{16.219} & \multirow{2}{*}{0.000} \\
\hline Yes & 29 & $7.86(4-15)$ & & \\
\hline \multicolumn{5}{|l|}{ Invasion } \\
\hline No & 16 & $15.75(9-24)$ & \multirow{2}{*}{32.271} & \multirow{2}{*}{0.000} \\
\hline Yes & 30 & $7.27(4-12)$ & & \\
\hline \multicolumn{5}{|l|}{ Surgery } \\
\hline Radical & 14 & $16.64(10-24)$ & \multirow{3}{*}{50.165} & \multirow{3}{*}{0.000} \\
\hline Palliative & 18 & $8.50(6-12)$ & & \\
\hline Biopsy & 14 & $6.00(4-8)$ & & \\
\hline \multicolumn{5}{|l|}{ ALDH1A3 } \\
\hline- & 30 & $11.67(6-24)$ & \multirow{2}{*}{7.914} & \multirow{2}{*}{0.005} \\
\hline+ & 16 & $7.50(4-15)$ & & \\
\hline \multicolumn{5}{|l|}{ GPX3 } \\
\hline- & 27 & $8.00(4-15)$ & 9.917 & 0.002 \\
\hline+ & 19 & $13.69(7-24)$ & & \\
\hline $\mathrm{AC}$ & & & & \\
\hline Differentiation & & & & \\
\hline Well & 27 & $15.07(5-24)$ & & \\
\hline Moderately & 25 & $10.60(4-24)$ & 32.501 & 0.000 \\
\hline Poorly & 28 & $6.68(3-14)$ & & \\
\hline Tumor mass size & & & & \\
\hline$\leq 3 \mathrm{~cm}$ & 50 & $13.70(6-24)$ & 68.283 & 0.000 \\
\hline$>3 \mathrm{~cm}$ & 30 & $5.80(3-10)$ & & \\
\hline TNM stage & & & & \\
\hline $\mathrm{I}+\mathrm{II}$ & 21 & $18.96(5-24)$ & & \\
\hline III & 38 & $9.29(6-15)$ & 105.825 & 0.000 \\
\hline IV & 21 & $5.14(3-7)$ & & \\
\hline Lymph node me & & & & \\
\hline No & 30 & $16.27(4-24)$ & 42.372 & 0.000 \\
\hline Yes & 50 & $7.42(3-14)$ & & \\
\hline Invasion & & & & \\
\hline No & 31 & $16.68(7-24)$ & 55.535 & 0.000 \\
\hline Yes & 49 & $6.98(3-11)$ & & \\
\hline
\end{tabular}


TABLe 2: Continued.

\begin{tabular}{cccc}
\hline C.P characteristics & Samples $(n)$ & Average survival (month) & Chi-square \\
\hline Surgery & & & $P$ value \\
Radical & 26 & $18.31(10-24)$ & 113.141 \\
Palliative & 28 & $8.64(6-11)$ & 0.000 \\
Biopsy & 26 & $5.42(3-9)$ & 25.229 \\
ALDH1A3 & 45 & $13.36(6-24)$ & 0.000 \\
- & 35 & $7.37(3-18)$ & 40.507 \\
+ & & & 0.000 \\
GPX3 & 42 & $7.05(3-15)$ & \\
- & 38 & $14.82(5-24)$ & \\
+ & & & \\
\hline
\end{tabular}

C.P: clinicopathological.

TABLE 3: Multivariate cox regression analysis of survival rate in SC/ASC and AC patients.

\begin{tabular}{|c|c|c|c|c|c|c|c|c|}
\hline \multirow{2}{*}{ Groups } & \multirow{2}{*}{ Factors } & \multirow{2}{*}{$\mathrm{RC}$} & \multirow{2}{*}{ SE } & \multirow{2}{*}{ Wald } & \multirow{2}{*}{$P$} & \multirow{2}{*}{$\mathrm{RR}$} & \multicolumn{2}{|c|}{ 95\% confidence interval } \\
\hline & & & & & & & Lower & Upper \\
\hline \multicolumn{9}{|l|}{ SC/ASC } \\
\hline Differentiation & Well/moderately/poorly & .785 & .350 & 5.030 & 0.025 & 2.192 & 1.104 & 4.354 \\
\hline Tumor mass size & $\leq 3 \mathrm{~cm} />3 \mathrm{~cm}$ & 2.760 & .790 & 12.206 & 0.000 & 15.800 & 3.359 & 74.321 \\
\hline TNM stage & $\mathrm{I}+\mathrm{II} / \mathrm{III} / \mathrm{IV}$ & 1.181 & .449 & 6.918 & 0.009 & 3.258 & 1.351 & 7.854 \\
\hline Lymph metastasis & No/yes & 1.107 & .530 & 4.363 & 0.037 & 3.025 & 1.071 & 8.549 \\
\hline Invasion & No/yes & 2.891 & .849 & 11.595 & 0.001 & 18.011 & 3.411 & 95.111 \\
\hline Surgery & Radical/palliative/biopsy & 1.203 & .543 & 4.908 & 0.027 & 3.330 & 1.149 & 9.653 \\
\hline ALDH1A3 & $-1+$ & 1.071 & .521 & 4.226 & 0.040 & 2.918 & 1.051 & 8.102 \\
\hline GPX3 & $-1+$ & -1.333 & .497 & 7.194 & 0.007 & .264 & .100 & .698 \\
\hline \multicolumn{9}{|l|}{$\mathrm{AC}$} \\
\hline Differentiation & Well/moderately/poorly & 1.055 & .505 & 4.364 & 0.037 & 2.872 & 1.067 & 7.728 \\
\hline Tumor mass size & $\leq 3 \mathrm{~cm} />3 \mathrm{~cm}$ & .928 & .455 & 4.160 & 0.041 & 2.529 & 1.037 & 6.171 \\
\hline TNM stage & $\mathrm{I}+\mathrm{II} / \mathrm{III} / \mathrm{IV}$ & .997 & .447 & 4.975 & 0.026 & 2.710 & 1.128 & 6.509 \\
\hline Lymph metastasis & No/yes & .947 & .416 & 5.182 & 0.023 & 2.849 & 1.141 & 5.826 \\
\hline Invasion & No/yes & 1.449 & .514 & 7.947 & 0.005 & 4.259 & 1.555 & 11.663 \\
\hline Surgery & Radical/palliative/biopsy & 1.911 & .432 & 19.568 & 0.000 & 6.760 & 2.899 & 15.764 \\
\hline ALDH1A3 & $-1+$ & .942 & .330 & 8.148 & 0.004 & 2.565 & 1.343 & 4.898 \\
\hline GPX3 & $-1+$ & -1.024 & .367 & 7.785 & 0.005 & 0.359 & .175 & .737 \\
\hline
\end{tabular}

RC: regression coefficients; SE: standard error; RR: relative risk.

can function as antioxidants. Therefore, the expression of ALDH1A3 and GPX3, two oxygen stress-related molecules, was investigated in this study. Previous studies have demonstrated that GPX3 is downregulated in a variety of tumors, such as prostate cancer, thyroid cancer, colorectal cancer, gastric cancer, and breast cancer $[15,16,18]$. However, the predictive role of GPX3 in the prognosis of these cancer patients has not been observed. Moreover, no molecular markers related to the aggressive biological characteristics or prognosis of SC/ASC have currently been identified. In this study, we demonstrated that loss of GPX3 expression was significantly associated with metastasis, invasion, TNM stage, and poor prognosis in both SC/ASC and AC patients. These observed correlations may possibly indicate that GPX3 is a tumor suppressor in gallbladder cancers. Increases in reactive oxygen species levels have been associated with tumor cell proliferation, genetic instability, and subsequent increases in invasion, angiogenesis, and drug resistance $[9,10]$. Although this study provided no evidence on whether the expression and/or activity of other antioxidant enzymes were decreased and whether the suppression of these enzymes plays a key role in the increased ROS levels in GBCs, the observed decrease in GPX3 expression suggested its involvement in oxidative stress.

Aldehyde dehydrogenases are known to modulate several cell functions, including proliferation, differentiation, and survival, as well as cellular response to oxidative stress [34]. In addition, cancer cell-acquired drug resistance has been demonstrated to be associated with the transcriptional activation of ALDH1 expression [35]. In contrast, ALDH1A1 and ALDH3A1 have been demonstrated to function as ocular antioxidants [20]. In this study, positive ALDH1A3 expression 


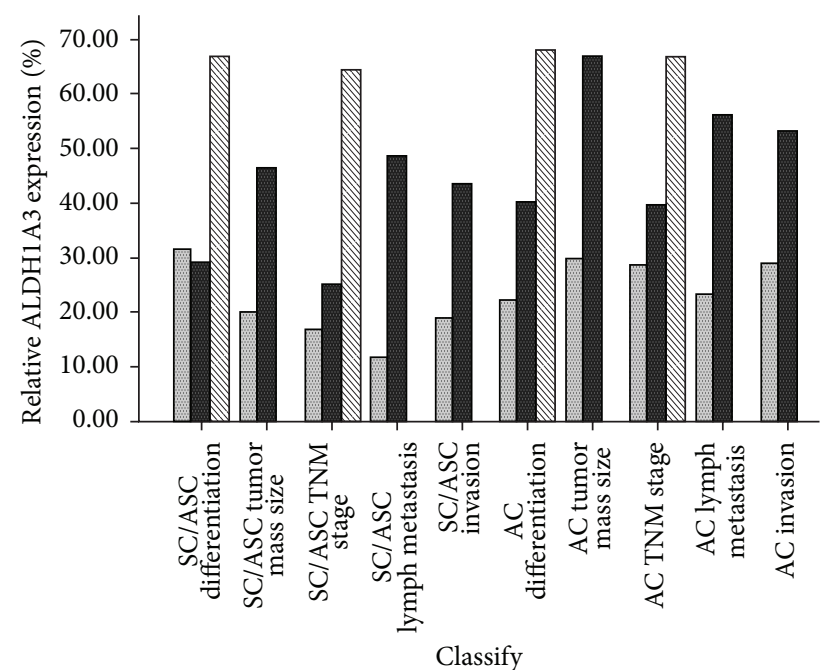

Clinicopathological characteristics

$\square \begin{array}{lll}1 & \square 3\end{array}$

圆 2

(a)

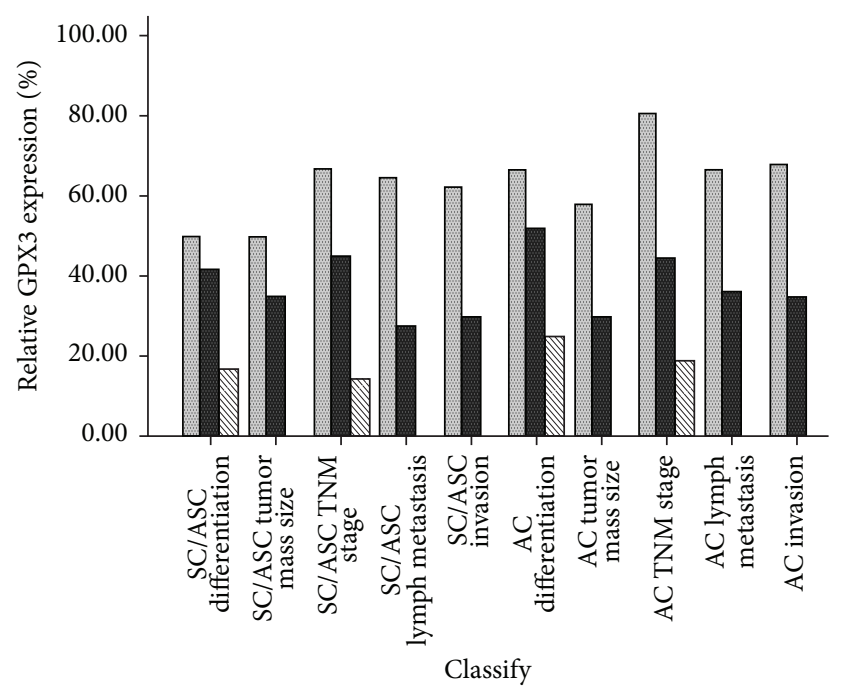

Clinicopathological characteristics

$\square \begin{array}{lll}\square & \square 3\end{array}$

욜 2

(b)

FIGURE 3: Semiquantitative analysis of ALDH1A3 and GPX3 expressions. (a), relative ALDH1A3 expression in SC/ASC and AC. (b), relative GPX3 expression in SC/ASC and AC. 1, 2, 3 represents the clinicopathological characteristics with same subclassification in Table 1.

in AC patients was associated with poor differentiation, large tumor size, high TNM stage, lymph node metastasis, and invasion. In SC/ASC patients, positive ALDH1A3 expression was also associated with high TNM stage, invasion, and lymph node metastasis. This suggests that ALDH1A3 in GBC plays a role in the proliferation, differentiation, and survival of tumor cells. In contrast, the possible antioxidant activities of ALDH1A3 have not been observed. In addition, both the $\mathrm{AC}$ and SC/ASC patients with positive ALDH1A3 expression

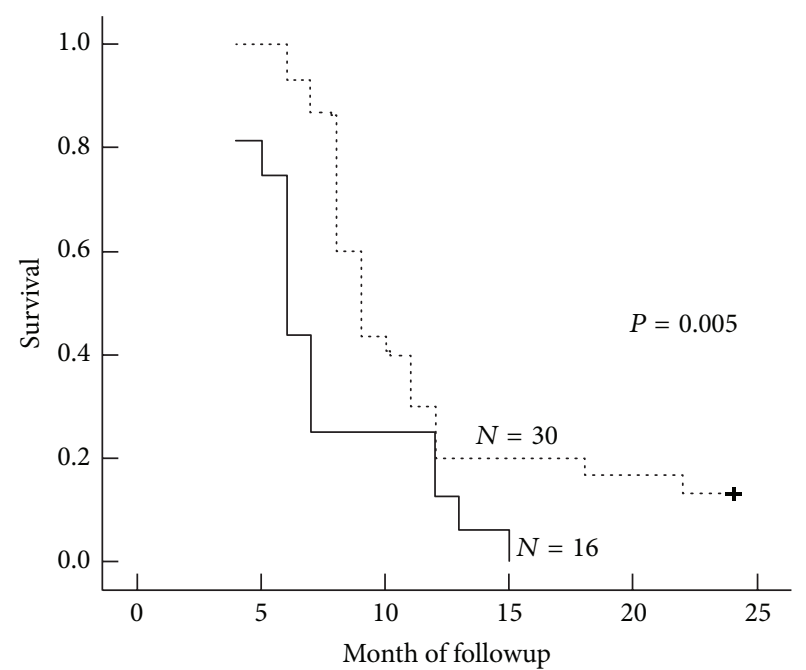

ALDH1A3
$\neg+$
$\cdots:-$
$+\quad--$ Censored

(a)

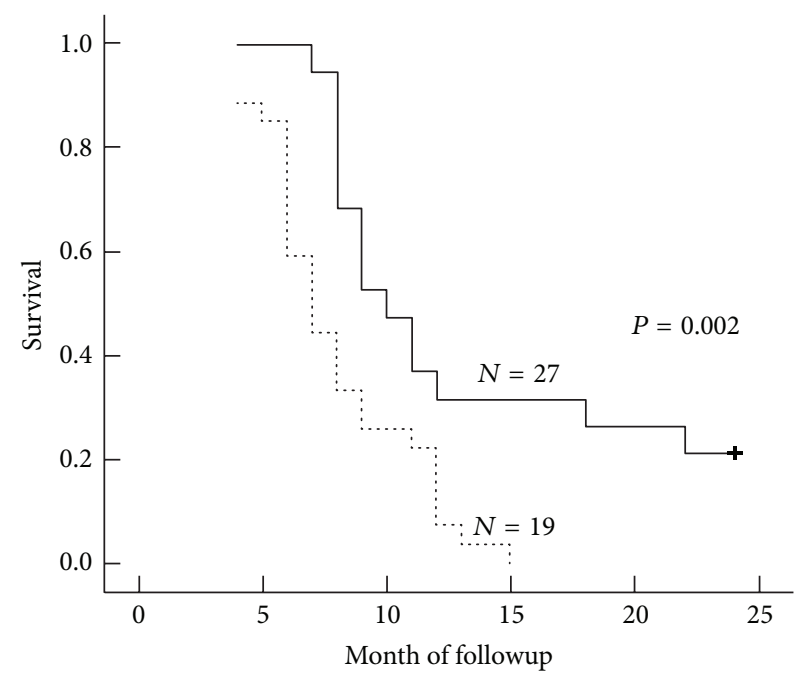

GPX3

$\neg+$

$\because \because-$

++- Censored

(b)

FIGURE 4: ALDH1A3 and GPX3 expressions and survival in patients with SC/ASC of gallbladder. (a), Kaplan-Meier plots of overall survival in patients with SC/ASC and with ALDH1A3 positive and negative expression. (b), Kaplan-Meier plots of overall survival in patients with SC/ASC and with GPX3 positive and negative expression.

exhibited poor survival. Recently, ALDH1 activity has been employed successfully as a stem cell marker in various cancers [35]. In addition, ALDH activity detected in tumor cells may actually be an indication of stem cells, because they are resistant to various chemotherapeutic drugs and linked to poor prognosis [22-25]. 


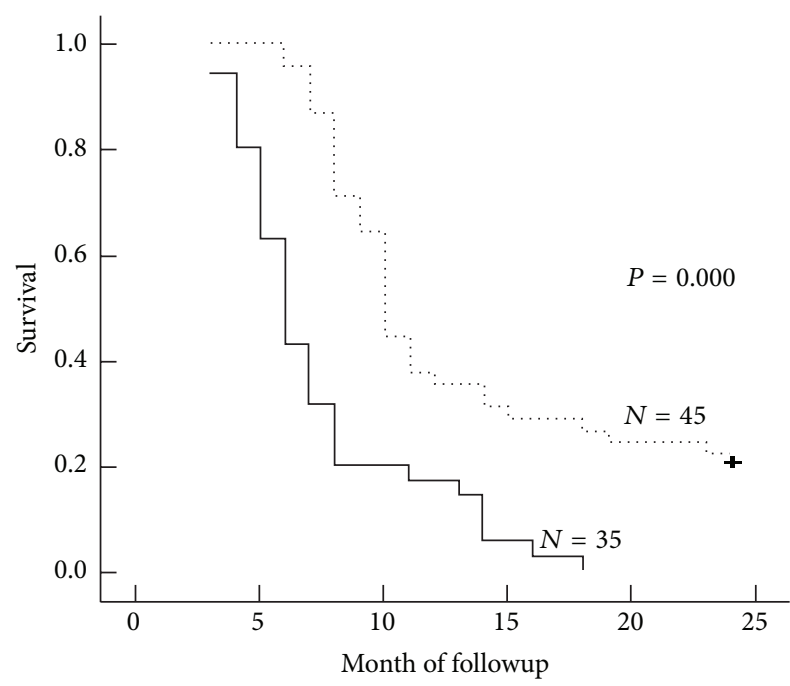

ALDH1A3

$\neg+$

$\because:-$

+-- Censored

(a)

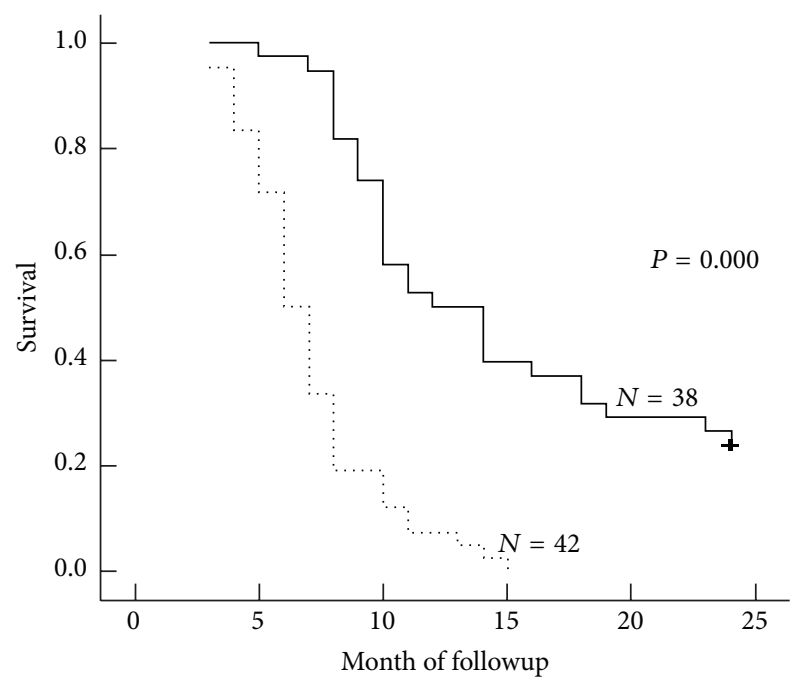

GPX3

$\neg+$

$\because:-$

++- Censored

(b)

Figure 5: ALDH1A3 and GPX3 expressions and survival in patients with AC of gallbladder. (a), Kaplan-Meier plots of overall survival in patients with $\mathrm{AC}$ and with ALDH1A3 positive and negative expression. (b), Kaplan-Meier plots of overall survival in patients with AC and with GPX3 positive and negative expression.

We acknowledge that although positive ALDH1A3 expression or negative GPX3 expression significantly correlated the shorter survival in both SC/ASC and AC patients, their regression coefficients or relative risk showed that the impact of positive ALDH1A3 expression or negative GPX3 expression on survival was small when compared with differences in tumor size, invasion, and differentiation. Therefore, ALDH1A3/GPX3 may be more effective as markers for proliferation and differentiation of gallbladder carcinoma than as tools for predicting overall survival. However, the real role of ALDH1A3/GPX3 on tumor growth and metastasis of gallbladder cancer should be investigated further in animal models using inhibitory agents of ALDH1, such as siRNA and/or overexpression of GPX3 gene. These preclinical studies may highlight pharmacologic targets for gene therapy of gallbladder cancer. The data from this study also raised questions on why do SC/ASC and AC share very similar gene expression profiles for ALDH1A3 and GPX3, and why the expression profiles of ALDH1A3 and GPX3, both molecules associated with oxidative stress, are closely related to TNM staging and metastasis.

In conclusion, the lowered expression of GPX3 and elevated expression of ALDH1A3 in AC and SC/ASC subtypes of GBC are important biological markers for the metastasis, invasion, and maybe prognosis of gallbladder cancer.

\section{Conflict of Interests}

All authors declared no conflict of interests.

\section{References}

[1] A. Jemal, R. Siegel, E. Ward et al., "Cancer statistics, 2008," CA Cancer Journal for Clinicians, vol. 58, no. 2, pp. 71-96, 2008.

[2] S. Jayaraman and W. R. Jarnagin, "Management of gallbladder cancer," Gastroenterology Clinics of North America, vol. 39, no. 2, pp. 331-342, 2010.

[3] W. G. Hawkins, R. P. DeMatteo, W. R. Jarnagin, L. Ben-Porat, L. $\mathrm{H}$. Blumgart, and Y. Fong, "Jaundice predicts advanced disease and early mortality in patients with gallbladder cancer," Annals of Surgical Oncology, vol. 11, no. 3, pp. 310-315, 2004.

[4] K. M. Reid, A. R. La Medina, and J. H. Donohue, "Diagnosis and surgical management of gallbladder cancer: a review," Journal of Gastrointestinal Surgery, vol. 11, no. 5, pp. 671-681, 2007.

[5] X. De Aretxabala, I. Roa, L. Burgos et al., "Gallbladder cancer: an analysis of a series of 139 patients with invasion restricted to the subserosal layer," Journal of Gastrointestinal Surgery, vol. 10, no. 2, pp. 186-192, 2006.

[6] T. Ootani, Y. Shirai, K. Tsukada, and T. Muto, "Relationship between gallbladder carcinoma and the segmental type of adenomyomatosis of the gallbladder," Cancer, vol. 69, pp. 26472652, 1992.

[7] J. C. Roa, O. Tapia, A. Cakir et al., "Squamous cell and adenosquamous carcinomas of the gallbladder: clinicopathological analysis of 34 cases identified in 606 carcinomas," Modern Pathology, vol. 24, no. 8, pp. 1069-1078, 2011.

[8] S. B. Park, Y. H. Kim, H. L. Rho, G. B. Chae, and S. K. Hong, "Primary carcinosarcoma of the gallbladder," Journal of the Korean Surgical Society, vol. 82, no. 1, pp. 54-58, 2012.

[9] M. B. Azad, Y. Chen, and S. B. Gibson, "Regulation of autophagy by reactive oxygen species (ROS): implications for cancer progression and treatment," Antioxidants and Redox Signaling, vol. 11, no. 4, pp. 777-790, 2009.

[10] H. Pelicano, D. Carney, and P. Huang, "ROS stress in cancer cells and therapeutic implications," Drug Resistance Updates, vol. 7, no. 2, pp. 97-110, 2004. 
[11] P. Storz, "Reactive oxygen species in tumor progression," Frontiers in Bioscience, vol. 10, no. 2, pp. 1881-1896, 2005.

[12] L. W. Oberley, "Mechanism of the tumor suppressive effect of MnSOD overexpression," Biomedicine and Pharmacotherapy, vol. 59, no. 4, pp. 143-148, 2005.

[13] H. Wiseman and B. Halliwell, "Damage to DNA by reactive oxygen and nitrogen species: role in inflammatory disease and progression to cancer," Biochemical Journal, vol. 313, no. 1, pp. 17-29, 1996

[14] T. L. Leto and M. Geiszt, "Role of Nox family NADPH oxidases in host defense," Antioxidants and Redox Signaling, vol. 8, no. 9-10, pp. 1549-1561, 2006.

[15] D. Agnani, O. Camacho-Vanegas, C. Camacho et al., "Decreased levels of serum glutathione peroxidase 3 are associated with papillary serous ovarian cancer and disease progression," Journal of Ovarian Research, vol. 4, no. 1, article 18, 2011.

[16] T. Paukert, R. Sailer, W. S. L. Strauss, M. Schubert-Zsilavecz, and A. Zimmer, "Glutathione peroxidase isoenzymes in human tumor cell lines," Pharmazie, vol. 66, no. 11, pp. 894-898, 2011.

[17] Y. Saga, M. Ohwada, M. Suzuki et al., "Glutathione peroxidase 3 is a candidate mechanism of anticancer drug resistance of ovarian clear cell adenocarcinoma," Oncology Reports, vol. 20, no. 6, pp. 1299-1303, 2008.

[18] R. Brigelius-Flohé and A. Kipp, "Glutathione peroxidases in different stages of carcinogenesis," Biochimica et Biophysica Acta, vol. 1790, no. 11, pp. 1555-1568, 2009.

[19] H. Riveros-Rosas, A. Julian-Sanchez, and E. Pina, "Enzymology of ethanol and acetaldehyde metabolism in mammals", Archives of Medical Research, vol. 28, no. 4, pp. 453-471, 1997.

[20] S. A. Marchitti, Y. Chen, D. C. Thompson, and V. Vasiliou, "Ultraviolet radiation: cellular antioxidant response and the role of ocular aldehyde dehydrogenase enzymes," Eye and Contact Lens, vol. 37, no. 4, pp. 206-213, 2011.

[21] P. Marcato, C. A. Dean, C. A. Giacomantonio, and P. W. K. Lee, "Aldehyde dehydrogenase its role as a cancer stem cell marker comes down to the specific isoform," Cell Cycle, vol. 10, no. 9, pp. 1378-1384, 2011.

[22] D. M. Benson Jr., K. Panzner, M. Hamadani et al., "Effects of induction with novel agents versus conventional chemotherapy on mobilization and autologous stem cell transplant outcomes in multiple myeloma," Leukemia and Lymphoma, vol. 51, no. 2, pp. 243-251, 2010.

[23] N. E. Sládek, R. Kollander, L. Sreerama, and D. T. Kiang, "Cellular levels of aldehyde dehydrogenases (ALDH1A1 and ALDH3A1) as predictors of therapeutic responses to cyclophosphamide-based chemotherapy of breast cancer: a retrospective study," Cancer Chemotherapy and Pharmacology, vol. 49, no. 4, pp. 309-321, 2002.

[24] J. Douville, R. Beaulieu, and D. Balicki, "ALDH1 as a functional marker of cancer stem and progenitor cells," Stem Cells and Development, vol. 18, no. 1, pp. 17-26, 2009.

[25] I. Ma and A. L. Allan, "The role of human aldehyde dehydrogenase in normal and cancer stem cells," Stem Cell Reviews and Reports, vol. 7, no. 2, pp. 292-306, 2011.

[26] T. Li, Y. Su, Y. Mei et al., "ALDH1A1 is a marker for malignant prostate stem cells and predictor of prostate cancer patients outcome," Laboratory Investigation, vol. 90, no. 2, pp. 234-244, 2010.

[27] G. Raz, K. E. Allen, C. Kingsley et al., "Hedgehog signaling pathway molecules and ALDH1A1 expression in early-stage non-small cell lung cancer," Lung Cancer, vol. 76, no. 2, pp. 191196, 2012.
[28] P. Marcato, C. A. Dean, P. Da et al., "Aldehyde dehydrogenase activity of breast cancer stem cells is primarily due to isoform ALDH1A3 and its expression is predictive of metastasis," Stem Cells, vol. 29, no. 1, pp. 32-45, 2011.

[29] D. Liu, Z. Yang, and S. Jiang, "Identification of musashi-1 and ALDH1 as carcinogenesis, progression, and poor-prognosis related biomarkers for gallbladder adenocarcinoma," Cancer Biomarkers, vol. 8, no. 3, pp. 113-121, 2010.

[30] Y. Zhang, X. Zhang, Z. N. Rabbani, I. L. Jackson, and Z. Vujaskovic, "Oxidative stress mediates radiation lung injury by inducing apoptosis," International Journal of Radiation Oncology, Biology, Physics, vol. 83, pp. 740-748, 2012.

[31] K. Nishihara, E. Nagai, Y. Izumi, K. Yamaguchi, and M. Tsuneyoshi, "Adenosquamous carcinoma of the gallbladder: a clinicopathological, immunohistochemical and flowcytometric study of twenty cases," Japanese Journal of Cancer Research, vol. 85, no. 4, pp. 389-399, 1994.

[32] M. Kondo, K. Dono, M. Sakon et al., "Adenosquamous carcinoma of the gallbladder," Hepato-Gastroenterology, vol. 49, no. 47, pp. 1230-1234, 2002.

[33] T. Shibata, A. Kokubu, M. Gotoh et al., "Genetic alteration of Keap1 confers constitutive Nrf2 activation and resistance to chemotherapy in gallbladder Cancer," Gastroenterology, vol. 135, no. 4, pp. 1358-1368, 2008.

[34] G. Muzio, M. Maggiora, E. Paiuzzi, M. Oraldi, and R. A. Canuto, "Aldehyde dehydrogenases and cell proliferation," Free Radical Biology and Medicine, vol. 52, no. 4, pp. 735-746, 2012.

[35] A. Yoshida, V. Dave, H. Han, and K. J. Scanlon, "Enhanced transcription of the cytosolic ALDH gene in cyclophosphamide resistant human carcinoma cells," Advances in Experimental Medicine and Biology, vol. 328, pp. 63-72, 1993. 


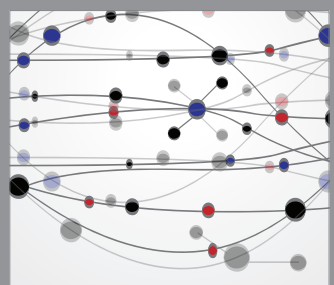

The Scientific World Journal
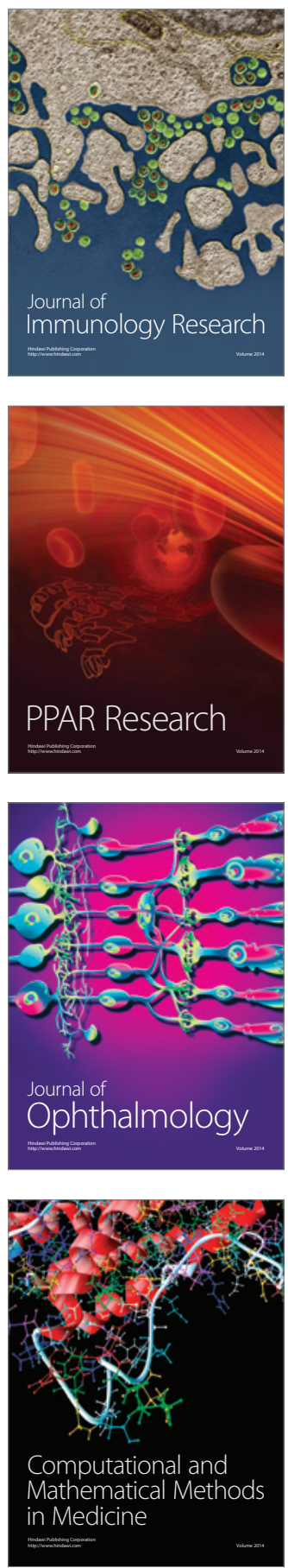

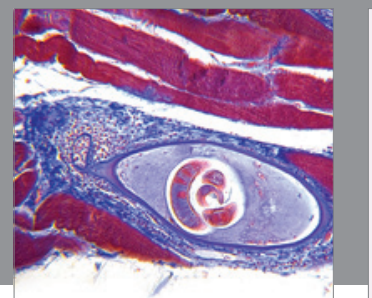

Gastroenterology

Research and Practice
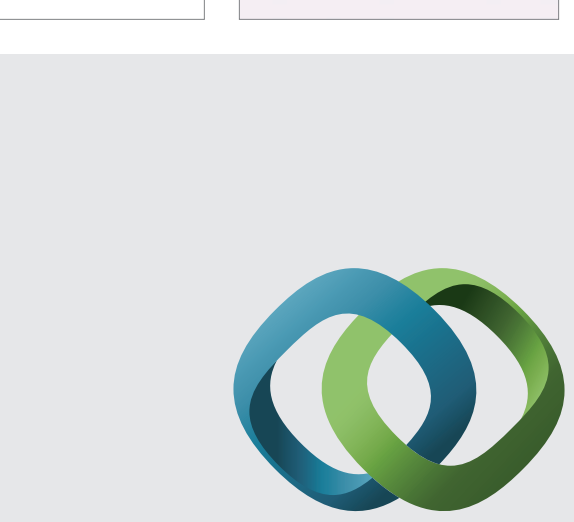

\section{Hindawi}

Submit your manuscripts at

http://www.hindawi.com
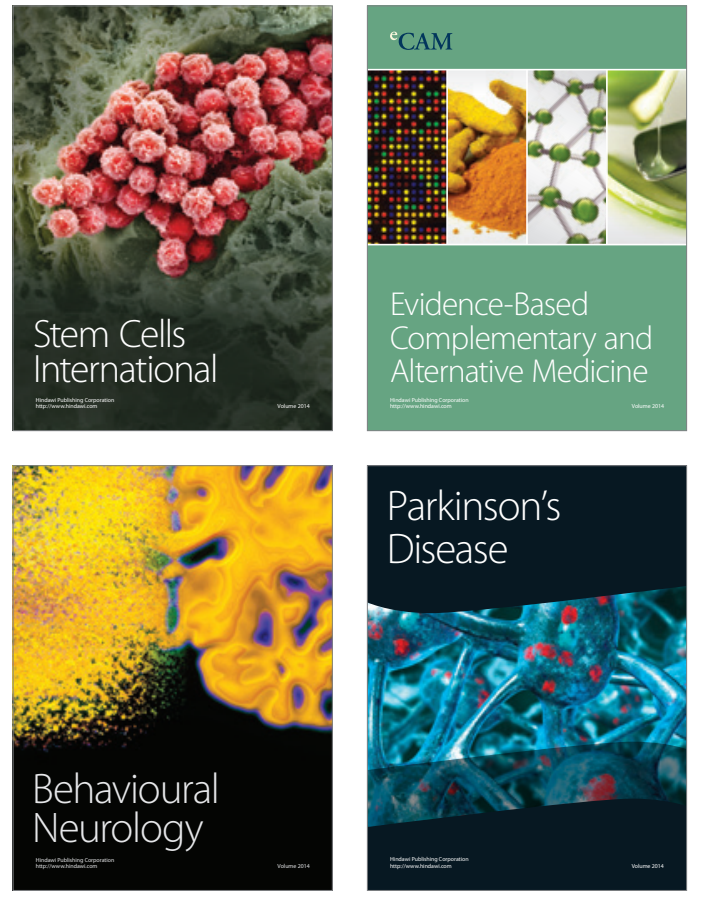
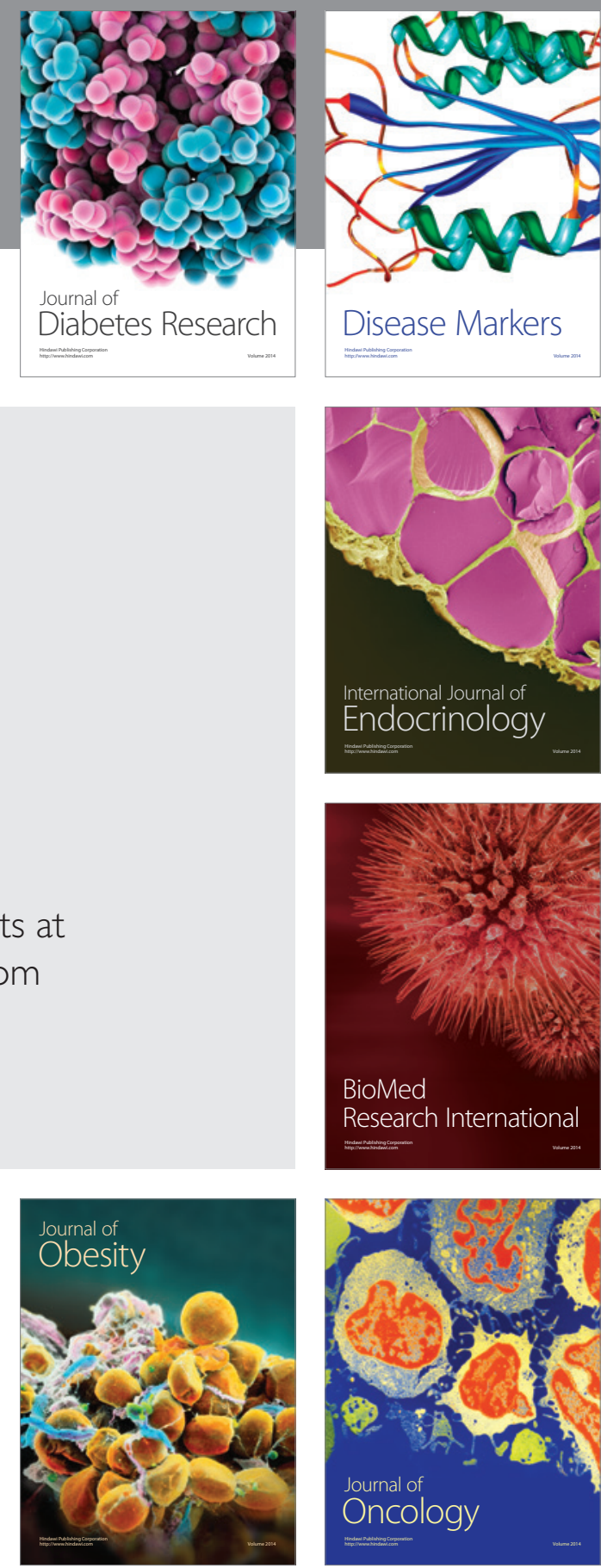

Disease Markers
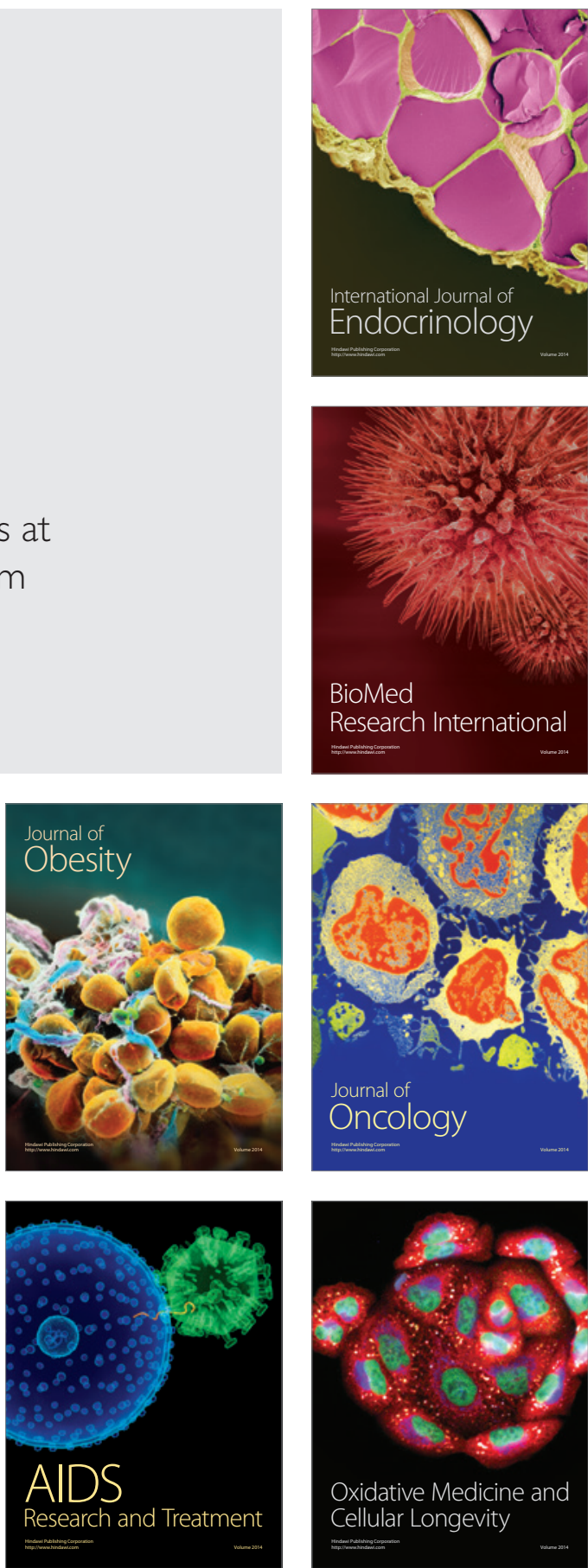